\title{
Etude morphologique de la phase mycélienne du Sporotrichum schenckii
}

\author{
(Hetkoen et Perkins, 1900) en microscopie photonique
}

\author{
par Monique THIBAUT, \\ Laboratoire de Parasitologie et de Mycologie (Pr M. LARIVIÈRE) \\ U.E.R. Cordeliers, 15, rue de l'Ecole de Médecine, F 75 -Paris $\left(6^{\circ}\right)$
}

\begin{abstract}
Résumé
Sur milieu de Sabouraud gélosé glucosé, à la température du laboratoire, la croissance du Sporotrichum schenckii (Hetkoen et Perkins, 1900) est visible en trois à quatre jours. Les colonies sont d'abord petites et blanches. Lorsque la croissance commence, la surface de la colonie devient humide, plissée et membraneuse. La couleur peut varier du crème au noir. Microscopiquement, les hyphes délicates, ramifiées et septées portent des conidies latéralement ou en bouquets terminaux sur les rameaux latéraux.
\end{abstract}

\section{Summary}

On Sabouraud's glucose agar, at room temperature, the growth of Sporotrichum schenckii (Hetkoen and Perkins, 1900) is recognizable within three to four days. At first, the colonies are small and white. As growth increases, the surface of the colony becomes moist, wrinkled and membranous. The color may vary from cream to black. Microscopically, the delicate, branching, septate hyphae bear conidia laterally or in groups from the ends of lateral branches.

Le Sporotrichum schenckii se présente, en vie saprophytique normale et dans les cultures, sous la forme mycélienne. Ce champignon dimorphique, potentiellement pathogène pour l'homme et certains animaux, n'est habituellement pas visible à l'examen microscopique direct du matériel pathologique. Par contre, sa culture est toujours facile. Le but de ce travail est de montrer la morphologie macroscopique et 
microscopique de la phase mycélienne. Dans une précédente publication (M. Thibaut 1970) nous avions étudié son dimorphisme et les facteurs permettant la transformation de la forme mycélienne en forme levure.

\section{Observations personnelles.}

\section{A. - Morphologie macroscopique (photo 1).}

Ensemencé en tubes ou en boîtes de Pétri, sur milieu gélosé glucosé de Sabouraud ou sur milieu au moût de bière gélosé, à la température de $20^{\circ}$, le Sporotrichum schenkii forme des colonies, visibles à l'œil nu à partir du quatrième jour. A ce stade, les cultures sont petites, de couleur blanche ou crémeuse; elles sont humides et brillantes; leur consistance est déjà élastique. Au fur et à mesure que la culture vieillit, elle s'étend; sa surface reste brillante, elle est d'abord lisse puis se plisse; elle est plus irrégulière au centre que sur les bords. Les sillons formés entre les plis sont dans leur ensemble toujours radiaires. La consistance de la culture devient plus ferme et plus caoutchouteuse avec l'âge. Sa couleur devient plus ou moins ocre; elle varie de celle du miel à celle des noisettes, mais dans certaines souches, elle peut être brune, brun-noir ou noire. Dans les très vieilles cultures, les colonies sont hérissées de mèches, ou de corémies fertiles.

\section{B. - Morphologie microscopique.}

Nous avons étudié la morphologie de la phase mycélienne, en microscopie optique, sur des cultures sur lames âgées de 2 à 50 jours. Nous les avons examinées, soit à l'état frais, soit après fixations et colorations diverses. Le mycélium est rampant, très fin et délicat (photo 2) : $1 \mu$ de diamètre, quelquefois moins, rarement plus. Il est cloisonné, incolore, abondamment ramifié et enchevêtré. Les conidies sont, soit disposées en manchons autour des filaments et insérées sur ceux-ci par l'intermédiaire de stérigmates, soit réunies au sommet d'un filament couché sur l'extrémité duquel elles forment alors un bouquet caractéristique (photo 3). Les jeunes spores sont hyalines ; les spores adultes sont plus ou moins pigmentées.

\section{A. - Septation des hyphes.}

Les hyphes sont cloisonnées assez irrégulièrement, de sorte que leurs articles sont inégaux : la longueur de ceux-ci varie de 10 à $40 \mu$. Les septa sont perpendiculaires au grand axe des hyphes.

\section{B. - Dimensions.}

Le diamètre des filaments mycéliens est extrêmement petit ; $1 \mu$ et même souvent moins. Il n'est pas constant tout au long d'un même filament; il est parfois un peu plus large au niveau des septa. D'autre part, les hyphes à conidies terminales vont en s'effilant ou en se renflant en vésicule. 


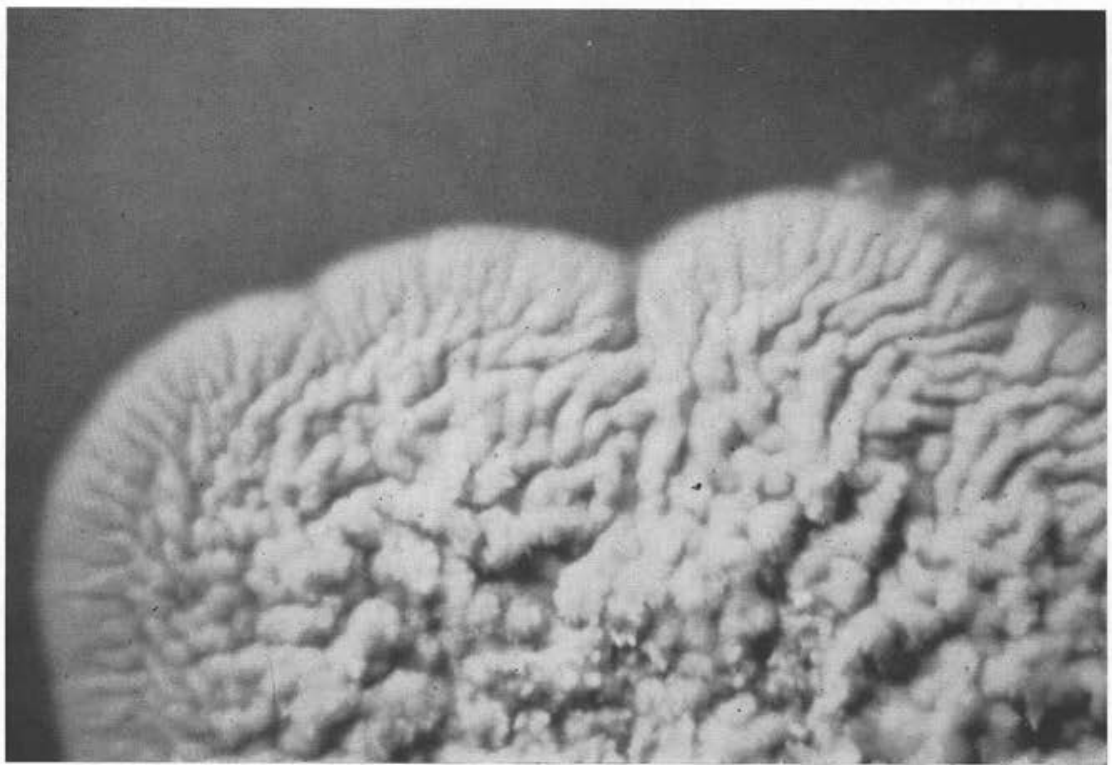

Рното 1. - Aspect macroscopique d'une culture du Sporotrichum schenckii sur milieu de Sabouraud. $\times 8$

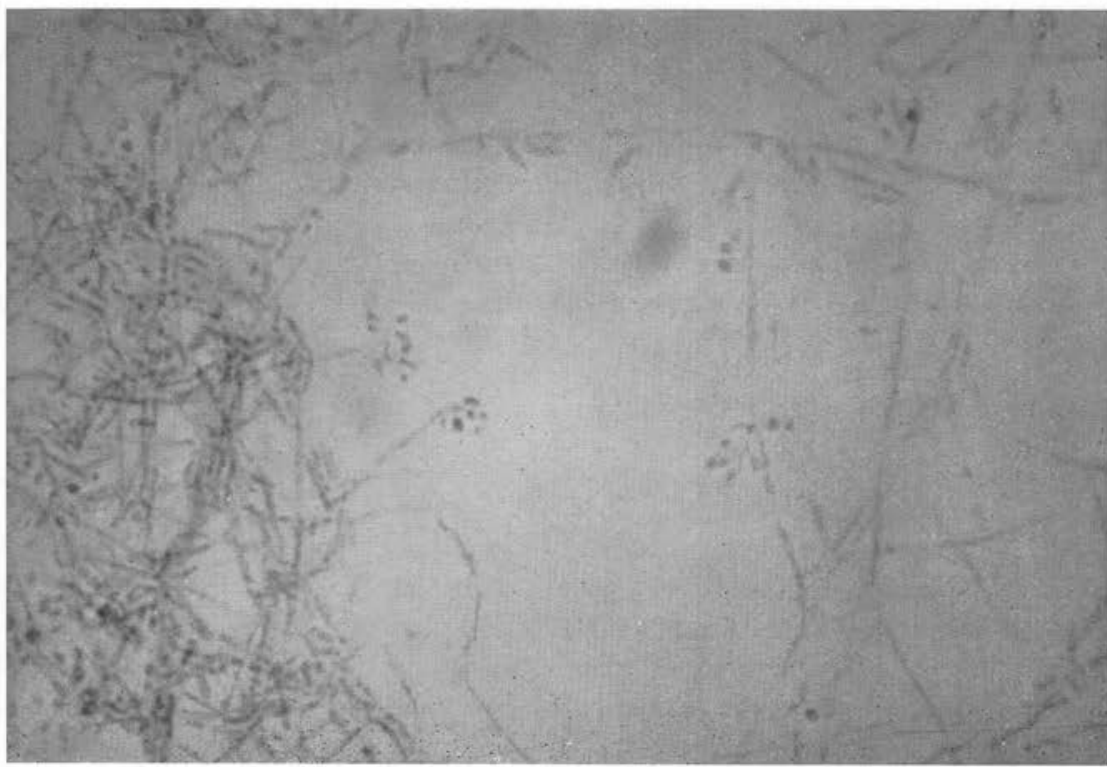

Pното 2. - Sporotrichum schenckii. Culture sur lame. Coloration: bleu de méthylène. $\times 330$ 


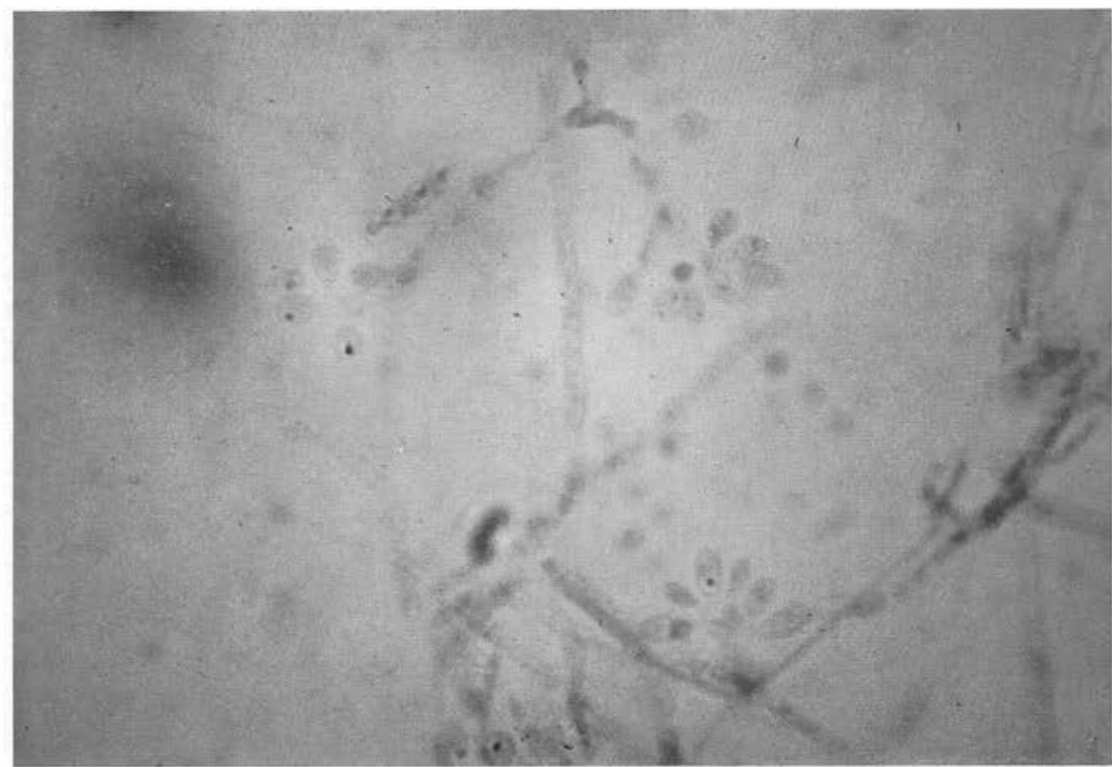

Fното 3. - Sporotrichum schenckii. Culture sur lame. Coloration: bleu de méthylène. Bouquets de conidies en pétale de rose. $\times 1.666$

\section{C. - Ramifications et anastomoses.}

Les ramifications sont abondantes. Les anastomoses sont fréquentes; elles se produisent lorsque des filaments parviennent au contact l'un de l'autre. D'autre part, les filaments sont parfois agrégés; ils forment alors des mèches stériles ou des corémies conidifères les unes et les autres dressées. En outre, certains filaments ont tendance à former des boucles, ou même des vrilles à plusieurs spires, rappelant un peu le filament ascogonial qui préside à la formation du périthèce chez certains Ascomycètes.

\section{D. - LES CONIDIES.}

\section{$1^{\circ}$ Forme.}

Les conidies du Sporotrichum schenckii, sont des spores entières, non septées: ce sont des amérospores. Ces spores, asexuées et oblongues, sont soit assez courtes et en forme de pétale de rose (photos 3 et 4), soit allongées, ce qui les fait ressembler à la fleur ligulée d'une marguerite (photo 5). Elles sont inégales dans une même culture et parfois sur un même filament. On peut voir sur leur surface, au plus petit de leurs deux pôles, une petite surface non colorée qui représente leur point d'attache sur le filament générateur. 


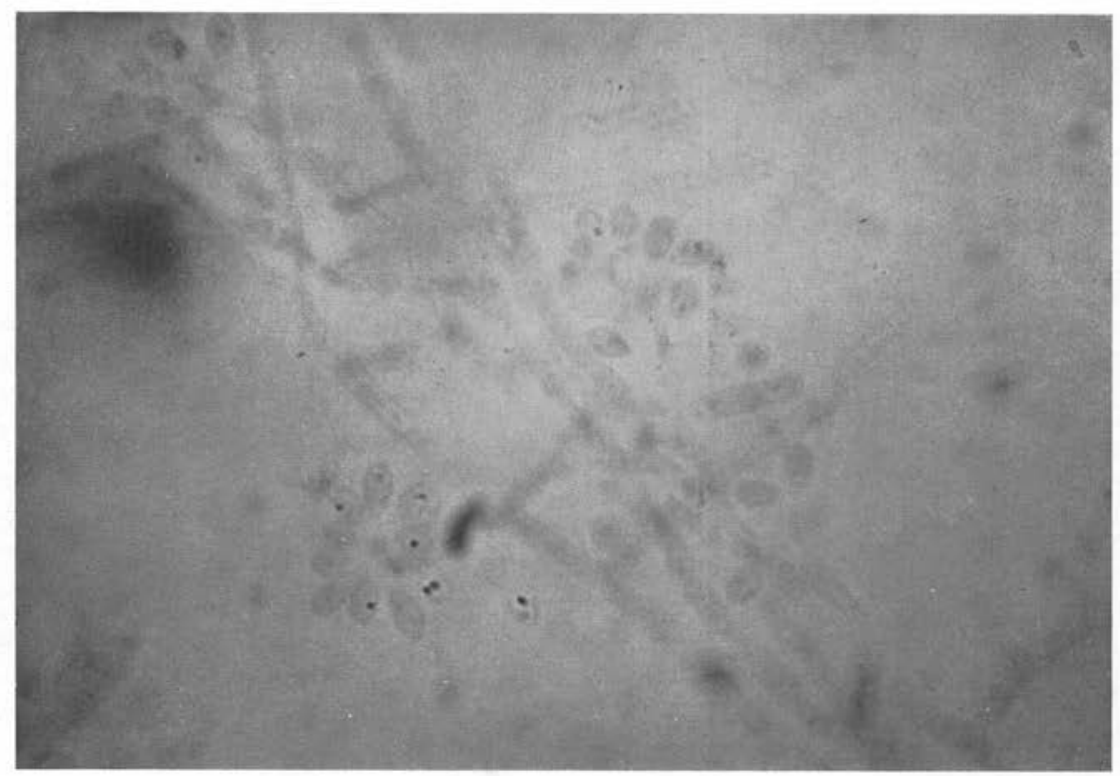

Рното 4. - Sporotrichum schenckii. Culture sur lame. Coloration: bleu de méthylène. Bouquets de conidies en pétale de rose. $\times 1.666$

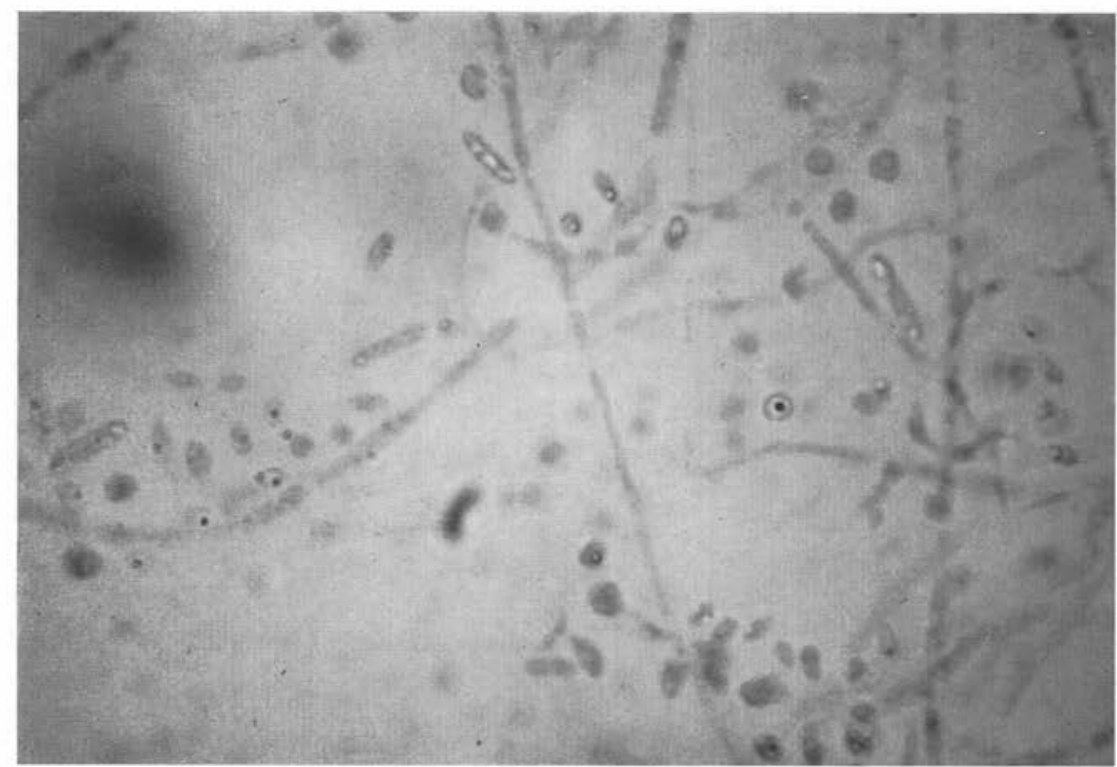

Рното 5. - Sporotrichum schenckii. Culiure sur lame. Coloration: bleu de méthylène. Conidies en pétale de rose et en ligule de marguerite. Chlamydospores. $\times 1.666$ 


\section{2: Dimensions.}

La taille des conidies varie. Elle est de :

- 2 à $3 \mu$ de long sur $1 \mu$ de large, pour les formes en pétale de rose ;

- 5 à $6 \mu$ de long sur 1 à $1,2 \mu$ de large, pour les formes en ligule de marguerite.

$3^{\circ}$ Mode d'insertion.

a) Insertion directe sur les filaments mycéliens.

Les conidies sont habituellement réunies au filament mycélien par un petit pédicelle ou stérigmate, mais elles peuvent aussi être sessiles. Le stérigmate est très court et pointu ; il mesure $0,5 \mu$ de large à sa base sur au plus $1 \mu$ de long. Lorsque les conidiospores portées par un stérigmate se détachent, la rupture se fait au sommet du stérigmate, qui subsiste sous la forme d'un denticule. Les portions de filaments mycéliens porteuses de tels denticules ont plus ou moins l'aspect d'une petite râpe, ou radule, de sorte que les conidies qu'ils portent sont des « radulaspores ».

Le long des hyphes, de telles radules sont souvent intercalaires, mais elles peuvent aussi être subterminales. Dans ce dernier cas, les stérigmates sont disposés sur deux rangs, en ordre distique, et d'autre part l'extrémité de l'hyphe s'effile et porte un bouquet de 3 ou 4 conidies, ce qui conduit au cas conidies disposées en bouquets sur des conidiophores.

b) Insertion sur des « conidiophores ».

Quand ceux-ci ne sont pas constitués par l'extrémité de radules subterminales, ils portent un bouquet de conidies plus nombreuses. Ils peuvent alors être, soit fortement arqués, avec les conidies sur leur bord convexe, soit vésiculeux, c'est-à-dire constitués par une petite vésicule qui, du moins dans certains cas, semble dériver d'un filament arqué, encore reconnaissable sur un de ses côtés (photo 4).

Quel que soit l'aspect du conidiophore, ses conidies peuvent être portées par des stérigmates, mais ceux-ci peuvent aussi faire défaut.

\section{E. - Les Chlamydospores (photo 5).}

$\mathrm{Au}$ fur et à mesure du vieillissement des cultures, apparaissent des chlamidospores. Ces spores de résistance sont plus volumineuses que les conidies. Elles sont arrondies ou ovoïdes, et entourées d'une paroi plus épaisse que celle des conidies formées de deux couches bien distinctes. Elles se colorent bieh plus intensément que les conidies par les colorants basiques. Elles sont intercalaires ou isolées et mesurent $3 \times 2 \mu$ lorsqu'elles sont ovoïdes, $4 \mu$ de diamètre lorsqu'elles sont rondes.

\section{Discussion.}

\section{A. - Morphologie macroscopique.}

La morphologie macroscopique du Sporotrichum schenckii présente parfois des variations importantes. Ce sont ces modifications que de Beurmann et Gougerot (1911-1912) groupaient sous la dénomination de «pléomorphisme macroscopique »: 
variation de teinte, pléomorphisme de forme et de plissement, formation de filaments agrégés faisant saillie à la surface des colonies, poudrage des colonies. En fait, le terme de pléomorphisme est mauvais et il aurait mieux valu parler de polymorphisme. De plus chez certaines souches, peuvent apparaître des variations sectorielles.

$\mathrm{Si}$ nous avons noté, suivant les souches, des variations de teinte, de forme, de plicature et de piquants, par contre, nous n'avons jamais observé l'apparition de variations sectorielles. D'autre part, nous n'avons pas remarqué que l'addition de thiamine au milieu de culture, favorise la pigmentation de celle-ci, ainsi que l'avait montré Reid (1952), mais il nous a semblé qu'elle donne aux cultures, un aspect plus tourmenté, plus godronné et qu'elle favorise la formation des mèches.

\section{B. - Morphologie microscopique.}

Certaines souches ne possèdent pas de radulaspores, mais seulement des bouquets de conidies. La sporulation en bouquet est caractéristique mais le conidiophore peut être long ou court et le nombre des conidies insérées portées par son sommet est variable. La forme des spores est elle aussi variable, comme nous l'avons dit plus haut.

Nous avons également observé des chlamydospores. Eles avaient échappé à Matruchot (1910) qui, à propos du Sporotrichum schenckii, écrivait : «Il n'y a pas de chlamydospores connues. "Par contre, chez le Sporotrichum beurmannii, qui est le Sporotrichum schenckii, de Beurmann et Gougerot (1911-1912) en avaient observé.

Au sujet des boucles et des vrilles que peuvent former les hyphes du Sporotrichum schenckii, rappelons que Matruchot et Dassonville (1889) ont comparé les vrilles des Trichophyton avec celles que produisent les hyphes péridiales du Ctenomyces serratus, et qu'ils ont alors suggéré de classer les Dermatophytes parmi les Gymnoascaceae. Mais cela ne suffit pas pour qu'on puisse considérer le Sporotrichum schenckii comme pouvant être, ou avoir été, la forme imparfaite d'une de celle-ci. Il diffère en effet des Gymnoascaceae par ses autres caractères, et des boucles ou des vrilles mycéliennes se forment chez des champignons n'ayant avec les Gymnoascaceae aucune proche parenté.

D'autre part, les vrilles formées par certaines hyphes du Sporotrichum schenckii évoquent les filaments ascogoniaux de divers Ascomycètes, comme par exemple les Ceratocystis. Aussi, Mariat et coll. (1968) ont-ils émis l'hypothèse que le Sporotrichum schenckii pourrait être la forme conidienne d'une espèce hétérothallique du genre Ceratocystis. Nous en avons discuté dans une autre publication sur la Taxinomie (1970).

\section{En Conclusion.}

Notre étude morphologique de la phase mycélienne du Sporotrichum schenckii, nous a montré les points caractéristiques suivants :

- Macroscopiquement, son polymorphisme en culture ; 
Microscopiquement,

- des hyphes dont le diamètre est extrêmement petit ;

- des articles très longs et de taille variable ;

- des « conidiophores » indifférenciés ou très peu différenciés d'avec le mycélium végétatif ;

- la disposition des conidies, soit sur des portions d'hyphes transformées en radules, soit en bouquets sur des conidiophores;

- des conidies du type amérosporé se présentant soit sous la forme en pétale de rose, soit sous la forme en ligule de marguerite.

\section{Bibliographie}

ANSEL (M.), 1957. - Mycoses et champignons parasites de l'homme, Do.n éd., Paris.

Ansel D'Imeux (M.), 1963. - Sporotrichose. Encyclopédie médico-chi.u gicale. Maladies infectieuses, 8124 A 10.

Brown (R.), Weintroub (D.) et Simpson (M. W.), 1947. - Timber as a source of sporotrichosis infection. Proceedings of the Transvaal mine medical officers' association, 5,33 .

De Beurmann (L.) et Gougerot (H.), 1911. - Les Sporotrichum pathogènes. Classification botanique. Arch. Parasitol., 15, 5-109.

— et —, 1912. — Les Sporotrichoses, Félix Alcan, éd., Paris.

Foulerton, 1900. - On the morphology and pathogenic action of Sporo:hrix schenckii, Transact. Path. Soc. London, p. 259.

Gougerot (H.) et De Beurmann (L.), 1908. - Découverte du Sporotichum beurmannii dans la natuie. Bull. et mém. Soc. méd. Hôp. Paris, 26, 733-738.

Hetroen (N.-L.) et Perkins (C.F.), 1900. - Refractory subcutaneous abcesses caused by Sporothrix schenckii, new pathogenic fungus. J. exptl. Med., 77-89.

Mariat (F.), Escudié (A.) et Gaxotre (P.), 1968. - Isolement de souches de Ceratocystis sp. à fo:me conidienne Sporotrichum de cuirs chevelus humains et de poils de rats. Comparaison avec l'espèce pathogène Sporotrichum schenckii. C.R. Acad. Sci., 267, 974-976.

Matruchot (L.), 1910. - Les champignons pathogènes, agents des sporotrichoses. C.R. Acad. Sci., 28 février 1910.

- et Ramond, 1905. - Un type nouveau de champignon pathogène chez l'homme. C.R. Séances Soc. Biol., 59, 379-380.

SCHENCK (B. R.), 1898. - On refractory subcutaneous abcesses caused by a fungus possibly related to the sporotrichia. Bull. Johns. Hopk. Hosp., 8, 286-290.

Thibaut (M.), 1970. - Biotopologie et dimorphisme du Sporotrichuin schenckii (Hetkoen et Perkins, 1900). Annales de Parasitologie Humaine et Comparée. 